\title{
El ataque contra una población civil en la práctica de la Corte Penal Internacional
}

\author{
Claudia Cárdenas Aravena*
}

\begin{abstract}
RESUMEN
El artículo muestra los criterios asumidos por la Corte Penal Internacional para determinar cuándo situaciones contemporáneas cumplen con los estándares para ser consideradas un ataque contra una población civil como elemento de contexto fundamental de los crimenes de lesa bumanidad. Se revisan las situaciones y casos relevantes que han sido conocidos por la Corte Penal Internacional desde su creación hasta agosto del 2020.
\end{abstract}

Ataque contra una población civil; Corte Penal Internacional; práctica en situaciones y casos

\section{The attack against a civilian population in the practice of the International Criminal Court}

\begin{abstract}
The article shows the criteria assumed by the International Criminal Court to understand when contemporary situations amount to an attack against a civilian population as fundamental element of crimes against humanity. The relevant case law of the International Criminal Court is reviewed from its creation until August of 2020.
\end{abstract}

Attack against a civilian population; International Criminal Court; case law

* Licenciada en Ciencias Jurídicas y Sociales, Universidad de Chile. Doctor iuris (Dr. iur), HumboldtUnversität zu Berlin, Alemania. Profesora del Departamento de Ciencias Penales de la Facultad de Derecho, Universidad de Chile. ORCID: https://orcid.org/https://orcid.org/0000-0002-8277-8523. Correo electrónico: ccardenas@derecho.uchile.cl

Artículo recibido el 3.3.20 y aceptado para su publicación el 9.10.20. 


\section{INTRODUCCIÓN}

$\mathrm{E}$ n todo el mundo somos testigos de crisis en las que surge la pregunta de si se han cometido crímenes de lesa humanidad. Y aunque en el derecho internacional existen descripciones típicas de aquello en que estos crímenes consisten ${ }^{1}$, cuando surge la necesidad de aplicar esas descripciones a hechos específicos es usual enfrentar dificultades. Parte de ellas derivan de que los contextos que enfrentamos usualmente difieren de las experiencias concretas ya pacíficamente aceptadas como contextos de crímenes de lesa humanidad durante el siglo pasado. En este escenario, para aplicar el derecho penal internacional a situaciones contemporáneas, resulta de ayuda conocer los criterios que la Corte Penal Internacional (CPI) viene aplicando desde su puesta en marcha, para un mejor entendimiento de cuándo una situación ${ }^{2}$ ha alcanzado el nivel de un ataque generalizado o sistemático contra una población civil como el elemento fundamental y necesario de los crímenes contra la humanidad.

Este artículo se centra en la práctica de la CPI en la determinación de cuándo existe un ataque relevante contra una población civil, para los efectos de determinar que han existido crímenes de lesa humanidad. Se da especial relevancia a la práctica judicial, teniendo en cuenta el rol que cumplen las resoluciones judiciales como medio para la determinación de reglas de derecho, junto con la doctrina ${ }^{3}$. En este caso, las reglas relevantes del Estatuto de Roma (ER) son las contenidas en el art 7.1 y 7.2.a) ER y de los Elementos de los Crímenes (EC) ${ }^{4}$ para el artículo 7, introducción pfo. 3, cuyo contenido se reseñará al inicio de cada apartado, en lo que sea pertinente.

La opción de dar cuenta solo del contenido de las resoluciones de la CPI considera - por una parte- que, si bien varios de los criterios que allí se expresan cuentan con un desarrollo en otras jurisdicciones, un estudio más amplio de cada uno de los aspectos del ataque desbordaría el formato de artículo5; y -por otra- que muchos Estados hispanoparlantes han tipificado en sus derechos internos los crímenes de lesa humanidad como una forma de implementar el ER ${ }^{6}$, guiándose para ello en parte importante por su texto, y cuando los elementos del delito están descritos como en ese tratado, de no aparecer que se les quiere dar un sentido diverso, resulta especialmente relevante conocer la práctica que la CPI -que además es el único tribunal penal internacional permanente- ha venido interpretando ese tratado.

${ }^{1}$ En 2017 la Comisión de Derecho Internacional de Naciones Unidas aprobó, en primera lectura, un "Texto del proyecto de artículos sobre los crímenes de lesa humanidad", que no contiene variaciones respecto del ER en la descripción del ataque.

${ }^{2}$ Acerca de situaciones y casos como términos técnicos, puede consultarse RASTAN, 2013, pp. 209 y ss.

${ }^{3}$ De acuerdo con derecho internacional, vid., a saber, el art. 38 del Estatuto de la Corte Internacional de Justicia.

${ }^{4}$ Cfr. arts. 9 y 21.1 ER.

${ }^{5}$ Se advierte que, cuando en una nota al pie se advierte que la fuente contiene más referencias, usualmente ellas conducen a resoluciones previas del Tribunal Penal Internacional para la ex-Yugoslavia (TPIY) o del Tribunal Penal Internacional para Ruanda.

${ }^{6}$ Una recopilación de las legislaciones de implementación se encuentra en www.legal-tools.org. 
Los aportes de este trabajo han de ser, en primer lugar, la sistematización actualizada de la práctica de la CPI en este ámbito y, en segundo lugar, su puesta a disposición en español, que siendo uno de los idiomas oficiales de la CPI no es -en cambio- uno de sus idiomas de trabajo, estando la mayoría de las resoluciones disponibles solo en inglés o en francés ${ }^{7}$. En tercer término, se espera que la provisión de ejemplos contemporáneos de ataques contra la población civil pueda colaborar en lograr una mejor comprensión de esta clase de crímenes. En todo caso, no ha de perderse de vista que la CPI está llamada a conocer de los crímenes más graves contra el derecho internacional (y por oposición no de todos los crímenes contra el derecho internacional), por lo que estos ejemplos lo serán siempre de situaciones especialmente graves, incluso dentro del ámbito del derecho penal internacional.

Para dotar de contenido a los apartados siguientes, se trabajó originalmente sobre la base de un corpus de 61 resoluciones de la CPI de los últimos 15 años, referidas a aquellos hitos procesales en los que resulta necesario algún grado de convicción respecto de la comisión de crímenes de lesa humanidad. De acuerdo con lo anterior, se revisaron las resoluciones que autorizan a la apertura de una investigación, cuando esto procede de acuerdo con art. 15 ER; las decisiones recaídas en solicitudes de la Fiscalía de acuerdo con art. 58 ER (orden de detención y entrega u orden de comparecencia); las resoluciones en las que se ordena la detención o la comparecencia de personas imputadas; las que ponen fin a la etapa de confirmación de cargos de acuerdo con art. 61 ER; las sentencias definitivas (art. $74 \mathrm{ER}$ ) y las resoluciones que han fallado apelaciones de algunas de las anteriores clases de resoluciones. Al revisar el corpus inicial se constató que no en todas las resoluciones se contenían desarrollos útiles al logro de los objetivos de este trabajo, por lo que en definitiva solo se usan como referencias del trabajo las 34 resoluciones de la CPI que se refieren expresamente a los elementos o a las características del ataque contra una población civil como requisitos de la imputación de crímenes de lesa humanidad, dictadas desde el inicio de su trabajo hasta agosto del 2020.

En texto comienza sistematizando los hallazgos relativos a los elementos del ataque (I.); y al carácter del ataque: generalizado o sistemático (II.). En cada nivel se examinarán los estándares o criterios abstractos, aplicables a todos los casos; además de ejemplos concretos de aplicación de esos estándares, que sirven para ilustrar hechos que se han interpretado como constitutivos de un ataque, pero no necesariamente dan cuenta de requisitos a cumplir extrapolables como tales a otros casos. En las referencias se procura seguir un orden cronológico, de modo que pueda apreciarse si existe alguna evolución. El texto termina con una valoración (III.) y conclusiones.

${ }^{7}$ Existe un trabajo anterior, publicado el 2013, también enfocado a la práctica de la CPI en este punto. BeLtrán, 2013, p. 49 y ss. Los años de práctica adicionales que alcanza a cubrir este estudio se estiman relevantes para establecer qué criterios se pueden estimar ya consolidados, y en cuáles se nota una variación. 


\section{El ataque}

El ataque contra una población civil está definido en el art. 7.2.a) ER, como "una línea de conducta que implique la comisión múltiple de actos mencionados en el párrafo 1 contra una población civil, de conformidad con la política de un Estado o de una organización de cometer ese ataque o para promover esa política”. Esto se replica, aunque no textualmente, en los EC para el art. 7, introducción pfo. 3.

En las resoluciones de la CPI se encuentra reiteradamente la afirmación de que el ataque puede entenderse como una campaña u operación dirigida contra una población civil $^{8}$.

A continuación se presenta separadamente la información respecto de la multiplicidad de actos del art. 7.1 ER (1.), lo relativo a una población civil como objeto del ataque (2.) y lo concerniente a la política que hace que estos actos sean inteligibles como una línea de conducta (3.).

\section{Sobre la comisión múltiple de actos del art. 7.1 ER}

De acuerdo con el art. 7.2 ER y a los EC para el art. 7, introducción pfo. 3, el ataque típico requiere la comisión múltiple de actos de los descritos en los literales del art. 7.1 $\mathrm{ER}^{9}$. Los EC agregan que no es necesario que los actos constituyan un ataque militar.

\section{a) Criterios abstractos en las resoluciones de la Corte Penal Internacional}

Se ha explicitado que los actos que constituyen el ataque pueden implicar cualquier forma de violencia contra una población civil ${ }^{10}$.

Respecto de la multiplicidad de actos a la que se refiere el ER, se ha explicado que implica un requisito cuantitativo, que es mayor a la existencia de "algunos" o "varios" $\operatorname{actos}^{11}$. Sin embargo, resulta notorio que no se suele trabajar con cifras al hacer la subsunción y, en general, la CPI es bastante cauta con su uso.

${ }^{8}$ CPI, 15.7.2009, N ${ }^{\circ}$ ICC-01/05-01/08, pfo. 75; CPI, 31.3.2010, No ICC-01/09, pfo. 80; CPI, 15.11.2011, N N $^{\circ}$ ICC-02/11, pfo. 31; CPI, 30.11.2011, No ICC-02/11-01/11, pfo. 30; CPI, 23.1.2012, No ICC-01/09-01/11, pfo. 164; CPI, 7.3.2014, , pfo. 1101; CPI, 12.6.2014, No ICC-02/11-01/11, pfo. 209; CPI, 21.3.2016, No ICC-01/05-01/08, pfo. 149; CPI, 9.11.2017, N ICC-01/17-X-9-US-Exp, 25 October 2017, pfo. 32; CPI, 22.5.2018, No ICC-01/12-01/18, pfos. 44 y 47 ; CPI, 8.11.2019, No ICC-01/12-01/18, pfo. 143; CPI, 14.11.2019, N ICC-01/19, pfo. 63.

${ }^{9}$ Se conocen con la denominación genérica de "actos inhumanos" y cuentan con elementos que ayudan a su determinación en el mismo tratado, en los EC, en la jurispruencia y en la doctrina del derecho penal internacional. Vid., a saber, Werle y Jessberger, 2017, p. 577 y ss. o Hall et al., 2017, pp. 178 y ss. con más antedentes.

${ }^{10}$ CPI, 7.3.2014, N ${ }^{o}$ ICC-01/04-01/07, pfo. 1101; CPI, 9.11.2017, No ICC-01/17-X-9-US-Exp, 25 October 2017, pfo. 32; CPI, 8.11.2019, Nº ICC-01/12-01/18, pfo. 144; CPI, 14.11.2019, No ICC-01/19, pfo. 63.

${ }^{11}$ CPI, 21.3.2016, No ICC-01/05-01/08, pfo. 150; CPI, 9.11.2017, No ICC-01/17-X-9-US-Exp, 25 October 2017, pfo. 32; CPI, 8.7.2019, No ICC-01/04-02/06, pfo. 663; CPI, 14.11.2019, N ICC-01/19, pfo. 63. Vid. asimismo CPI, 15.7.2009, N $\mathrm{N}^{\circ}$ ICC-01/05-01/08, pfo. 81, con más antecedentes. 
Resulta irrelevante la cantidad de actos de cada categoría el art. 7.1 ER que se incluyan en la imputación, siempre que cumulativamente satisfagan el umbral cuantitativo ${ }^{12}$.

Para probar la multiplicidad de actos, solo son relevantes aquellos enumerados en el art. 7.1 ER; sin perjuicio de que la prueba de otros actos pueda ser considerada para otros aspectos. Por ejemplo, pueden ayudar a determinar si el ataque fue dirigido contra una población civil, o se llevó adelante de conformidad con la política de un Estado u organización o para promoverlo, configurando una línea de conducta ${ }^{13}$.

\section{b) Situaciones y casos concretos}

- La situación de Libia habría involucrado actos de asesinato, encarcelamiento, tortura, persecución y otros actos inhumanos en varias localidades ${ }^{14}$.

- En la situación de Costa de Marfil, las fuerzas de Gbagbo habrían hecho uso excesivo de la fuerza y armamento pesado habría sido utilizado en lugares densamente poblados para dispersar a quienes apoyaban a Ouattara, traduciéndose ello en múltiples actos del $7.1 \mathrm{ER}^{15}$.

- En la situación de Burundi ya desde el primer día de protestas (26 de abril de 2015) miembros de la policía habrían disparado a civiles que se manifestaban, causando la muerte de algunos de ellos. En esta situación también se investigan detenciones arbitrarias, torturas, intentos de homicidio y homicidios ${ }^{16}$.

\section{Población civil como objeto del ataque}

Que el ataque ha de dirigirse contra una población civil es un requisito que se encuentra tanto en el art. 7.1 y 7.2 ER como en los EC para el art.7, introducción, pfo. 3.

\section{a) Criterios abstractos en las resoluciones de la Corte Penal Internacional}

Las resoluciones de la CPI han expresado que la voz población denota un colectivo, por oposición a un número limitado de individuos específicos ${ }^{17}$ o seleccionados al azar.

${ }^{12}$ CPI, 21.3.2016, N ICC-01/05-01/08, pfo. 150, con más referencias; CPI, 9.11.2017, N ${ }^{\circ}$ ICC01/17-X-9-US-Exp, 25 October 2017, pfo. 32; CPI, 14.11.2019, No ICC-01/19, pfo 63.

${ }^{13}$ CPI, 12.6.2014, N ${ }^{o}$ ICC-02/11-01/11, pfo. 210; CPI, 21.3.2016, No ICC-01/05-01/08, pfo. 151; CPI, 9.11.2017, No ICC-01/17-X-9-US-Exp, 25 October 2017, pfo. 32; CPI, 14.11.2019, No ICC-01/19, pfo. 63. Vid. asimismo CPI, 7.3.2014, No ICC-01/04-01/07, pfo. 1101; CPI, 8.7.2019, No ICC-01/0402/06, pfo. 663; CPI, 8.11.2019, No ICC-01/12-01/18, pfo. 143.

${ }^{14}$ CPI, 27.6.2011, No ICC-01/04-02/06, pfo. 31 ; CPI, 27.6.2011, No ICC-01/11, p. 4; CPI, 18.4.2013, $\mathrm{N}^{\circ}$ ICC-01/11-01/13, pfo. 5 y ss.

${ }^{15}$ CPI, 2.3.2012, No ICC-02/11-01/12, pfo. 22.

${ }^{16}$ CPI, 9.11.2017, No ICC-01/17-X-9-US-Exp, 25 October 2017, pfo. 35 y ss.

${ }^{17}$ CPI, 15.11.2011, No ICC-02/11, pfo. 32, con más antecedentes; CPI, 21.3.2016, No ICC-01/0501/08, pfos. 152, 154; CPI, 8.7.2019, No ICC-01/04-02/06, pfo. 667; CPI, 9.11.2017, No ICC-01/17-X9-US-Exp, 25 October 2017, pfo. 32; CPI, 14.11.2019, No ICC-01/19, pfo. 63. 
Además, se ha puesto de relieve que el uso de la palabra "una" indica que el art. 7.1 ER no debiera entenderse en el sentido de estar circunscrito a poblaciones definidas por factores distintivos de los más tradicionalmente asentados como factores de discriminación antijurídica, sino que se incluye a grupos de personas que se distinguen del resto de la población por otros factores, incluyendo su afiliación política (percibida) ${ }^{18}$. Tampoco se necesita probar que toda la población dentro de un área geográfica determinada ha sido objeto del ataque para entender que una población sí lo ha sido ${ }^{19}$.

Se ha reiterado que la población civil tiene que ser el objeto primario del ataque, por oposición a uno incidental ${ }^{20}$.

Por la naturaleza de la mayoría de las situaciones que ha conocido la CPI, en las resoluciones el carácter civil de la población se suele problematizar refiriéndose a contextos en los que ha existido un conflicto armado, señalándose que pueden aplicarse los criterios del derecho internacional humanitario, de acuerdo con ello ${ }^{21}$, durante los conflictos armados, ha de tratarse como civil a todo aquel que no conste que sea combatiente $^{22}$. Si un ataque contra una población civil se lleva a cabo durante un conflicto armado, en un área en la que hay civiles y combatientes legítimos, se acepta que víctimas de conductas concretas puedan no tener la calidad de civiles de acuerdo con el derecho internacional humanitario ${ }^{23}$. Factores relevantes para determinar si el ataque se dirigía

${ }^{18}$ CPI, 30.9.2008, No ICC-01/04-01/07, pfo. 399; CPI, 15.7.2009, No ICC-01/05-01/08, pfo. 76; CPI, 31.3.2010, N ICC-01/09, pfo. 81; CPI, 15.11.2011, No ICC-02/11, pfo. 32; CPI, 30.11.2011, N ICC02/11-01/11, pfo. 30 ; CPI, 23.1.2012, No ICC-01/09-02/11, pfo. 110; CPI, 23.1.2012, No ICC-01/0901/11, pfo. 164; CPI, 7.3.2014, No ICC-01/04-01/07, pfo. 1103; CPI, 12.6.2014, No ICC-02/11-01/11,

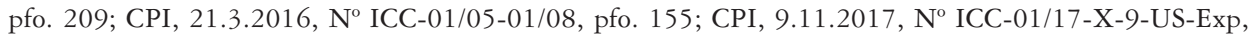
25 October 2017, pfo. 32; CPI, 22.5.2018, No ICC-01/12-01/18, pfo. 45 ; CPI, 8.7.2019, N ICC-01/0402/06, pfo. 667; con más referencias; CPI, 14.11.2019, N ICC-01/19, pfo. 63.

19 CPI, 15.7.2009, No ICC-01/05-01/08, pfo. 77; con más referencias; CPI, 15.11.2011, N ICC02/11, pfo. 33, con más referencias; CPI, 23.1.2012, N ICC-01/09-02/11, pfo. 109; CPI, 21.3.2016, $\mathrm{N}^{\circ}$ ICC-01/05-01/08, pfo. 154; CPI, 9.11.2017, N ICC-01/17-X-9-US-Exp, 25 October 2017, pfo. 32; CPI, 14.11.2019, No ICC-01/19, pfo. 63.

${ }^{20}$ CPI, 15.7.2009, N ${ }^{\circ}$ ICC-01/05-01/08, pfo. 76; CPI, 15.11.2011, No ICC-02/11, pfo. 33, con más referencias; CPI, 30.11.2011, $\mathrm{N}^{\circ}$ ICC-02/11-01/11, pfo. 30 ; CPI, 23.1.2012, No ICC-01/09-02/11, pfo. 109; CPI,7.3.2014, No ICC-01/04-01/07, pfo. 1105, con más referencias; CPI, 21.3.2016, No ICC-01/05-01/08, pfo. 154; CPI, 22.5.2018, N ICC-01/12-01/18, pfo. 45 ; CPI, 8.7.2019, No ICC-01/04-02/06, pfo. 668; CPI, 9.11.2017, No ICC-01/17-X-9-US-Exp, 25 October 2017, pfo. 32; CPI, 14.11.2019, No ICC-01/19, pfo. 63. Crítico sobre este criterio (aplicado en otra jurisdicción) se ha mostrado EBOE-OSUJI, 2008, p. 118 y ss.

${ }^{21}$ Abogan por una definición que no dependa de esa rama del derecho, a saber, ALIJA FERNÁNDEZ/ SAURA ESTAPÀ, 2016, pp. 1 y ss.; SADAT, 2017, pp. 197 y ss.

${ }^{22}$ CPI, 15.7.2009, N ${ }^{\circ}$ ICC-01/05-01/08, pfo. 78; con más referencias; CPI, 15.11.2011, No ICC-02/11,

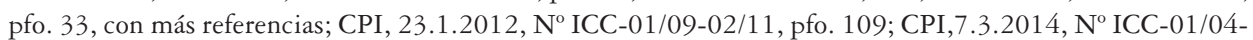
01/07, pfo. 1102; CPI, 21.3.2016, No ICC-01/05-01/08, pfo. 152; CPI, 9.11.2017, No ICC-01/17-X-9US-Exp, 25 October 2017, pfo. 32; CPI, 22.5.2018, No ICC-01/12-01/18, pfo. 45 ; CPI, 14.11.2019, No ICC-01/19, pfo. 63.

23 CPI, 21.3.2016, N ICC-01/05-01/08, pfo. 156; CPI, 8.7.2019, No ICC-01/04-02/06, pfo. 668; CPI, 9.11.2017, No ICC-01/17-X-9-US-Exp, 25 October 2017, pfo. 32; CPI, 14.11.2019, No ICC-01/19, pfo. 63. Una regla análoga está recogida, para el derecho internacional humanitario en conflictos armados internacionales, en el art. 50.3 del Protocolo Adicional I a los Convenios de Ginebra de 1949. 
contra una población civil -sin que se trate de un listado exhaustivo- incluyen los medios y métodos usados en el curso del ataque, el estatus y número de las víctimas, la naturaleza de los crímenes cometidos en el curso del ataque, las formas de resistencia al ataque durante su desarrollo y el grado de cumplimiento de las leyes de la guerra por parte de las fuerzas que llevaron adelante el ataque ${ }^{24}$.

\section{b) Situaciones y casos concretos}

A continuación se presentan ejemplos concretos de aplicación del derecho respecto del elemento "población civil”.

- En las situaciones de Darfur (Sudán) y de la República Centroafricana, la población civil objeto del ataque fue aquella que en la percepción de los atacantes habría estado vinculada con los rebeldes ${ }^{25}$ o se consideraba simpatizante respecto de ellos 26 .

- En la situación de Libia se ha considerado una población civil objeto del ataque a manifestantes o personas percibidas como disidentes políticos ${ }^{27}$.

- En las situaciones de Costa de Marfil y de Kenia se consideró que el ataque estuvo dirigido contra civiles a los que se consideraba partidarios de una persona ${ }^{28}$ o de un partido político ${ }^{29}$.

- En la situación de la República Democrática del Congo (RDC) se consideró como población civil objeto del ataque a la "no originaria" de Ituri ${ }^{30}$.

- En la situación concerniente a Burundi, la población civil objeto de ataque estaría conformada por opositores o personas consideradas desleales al Presidente, incluyendo a manifestantes, representantes de la sociedad civil, periodistas, etc. En esta situación, si bien se tiene en cuenta que las protestas no fueron enteramente pacíficas, se valora como un antecedente a favor de la existencia de un ataque contra una población civil el hecho de que miembros de la policía usaran municiones en respuesta a manifestantes que les tiraban piedras, y dispararan a civiles desarmados que escapaban de ellos, sin representar amenaza alguna. Se reconoce que tanto algunos manifestantes como personas no identificadas habrían estado envueltos en actos de violencia, que en algunos casos ocasionaron incluso muertes, y que, en algunos casos, las "Fuerzas de Seguridad" actuaron en respuesta a esos actos. Sin embargo, considerando los métodos utilizados por las "Fuerzas de Seguridad" y la

${ }^{24}$ CPI, 21.3.2016, N ICC-01/05-01/08, pfo. 153, con más antecedentes; CPI, 9.11.2017, Nº ICC01/17-X-9-US-Exp, 25 October 2017, pfo. 32; CPI, 14.11.2019, No ICC-01/19, pfo. 63.

${ }^{25}$ CPI, 27.4.2007, N ${ }^{\circ}$ ICC-02/05-01/07, pfo. 67; CPI, 1.3.2012, No ICC-02/05-01/12, pfo. 18.

${ }^{26}$ CPI, 10.6.2008, No ICC-01/05-01/08, pfos. 34-35.

${ }^{27}$ CPI, 27.6.2011, N ICC-01/04-02/06, pfo. 32 ; CPI, 27.6.2011, Nº ICC-01/11, p. 5.

${ }^{28}$ CPI, 30.11.2011, No ICC-02/11-01/11, pfo. 36 ; CPI, 12.6.2014, No ICC-02/11-01/11, pfos. 211, 225.

${ }^{29}$ CPI, 23.1.2012, No ICC-01/09-02/11, pfo. 142.

${ }^{30}$ CPI, 13.7.2012, No ICC-01/04-02/06, pfo. 27. 
clase de resistencia que encontraron, la naturaleza discriminatoria de los crímenes y el número de víctimas, la Sala estimó que había antecedentes para investigar un ataque dirigido contra una población civil ${ }^{31}$.

- En la situación de Mali se ha considerado como "una población civil" la población de una ciudad (Tombuktú) y sus alrededores ${ }^{32}$.

- En la situación de Afganistán, se entendió que la población civil objeto de ataque por los talibanes y otros grupos armados contrarios al gobierno estaría compuesta por quienes estos grupos consideraban opositores a sus mandatos e ideología, o que en su percepción prestaban apoyo al gobierno o a entidades extranjeras ${ }^{33}$.

\section{Política, su origen y la relación entre política y los actos del 7.1 ER}

De acuerdo con el art. 7.2 ER, los actos que conforman el ataque han de constituir una línea de conducta "de conformidad con la política de un Estado o de una organización de cometer ese ataque o para promover esa política”. Esa idea es replicada en los EC para el art. 7, introducción, pfo. 3, agregándose allí información acerca de cómo ha de manifestarse la política, explicitarse que "la 'política de cometer ese ataque' requiere que el Estado o la organización promueva o aliente activamente un ataque", y que "en circunstancias excepcionales, podría ejecutarse por medio de una omisión deliberada de actuar y que apuntase conscientemente a alentar un ataque de ese tipo", lo que "no se puede deducir exclusivamente de la falta de acción del gobierno o la organización”.

\section{a) Criterios abstractos en las resoluciones de la Corte Penal Internacional}

A continuación se entrega información diferenciada respecto de los hallazgos relativos a las características generales de la política, su origen y la relación con los actos inhumanos.

i) La política

Hay consenso en que la política no requiere ser adoptada formalmente, y para probarla se puede recurrir a indicios ${ }^{34}$.

Se ha señalado reiteradamente que un ataque que es planificado, dirigido u organizado - por oposición a actos de violencia aislados o espontáneos- satisfará la existencia

\footnotetext{
${ }^{31}$ CPI, 9.11.2017, No ICC-01/17-X-9-US-Exp, 25 October 2017, pfo. 35 y ss.

32 CPI, 22.5.2018, No ICC-01/12-01/18, pfo. 62 ; CPI, 8.11.2019, No ICC-01/12-01/18, pfo. 344

33 CPI, 12.4.2019, N ${ }^{\circ}$ ICC-02/17, pfo. 64.

${ }^{34}$ CPI, 30.9.2008, N ICC-01/04-01/07, pfo. 396 ; CPI, 31.3.2010, No ICC-01/09, pfo. 84, 85, 87 ;
} 15.11.2011, No ICC-02/11, pfo. 43; CPI, 30.11.2011, No ICC-02/11-01/11, pfo. 37; CPI, 13.7.2012, No ICC-01/04-02/06, pfo. 24 ; CPI, 12.6.2014, N ICC-02/11-01/11, pfo. 215; CPI, 21.3.2016, N ICC01/05-01/08, pfo. 160; CPI, 8.7.2019, No ICC-01/04-02/06, pfo. 674. 
de una política ${ }^{35}$; pero también se ha mencionado que la planificación o dirección serían solo indicios de que existe una política ${ }^{36}$. Algo análogo ocurre con el hecho de que se siga un patrón regular de conducta y que se empleen recursos públicos o privados. En varias resoluciones se menciona a estos factores como un requisito para que exista una política relevante ${ }^{37}$, en tanto que en otras -más recientes- se los alude como un indicio (entre otros posibles) de su existencia ${ }^{38}$. Se han referido además otros indicios útiles para establecer la existencia de una política, sin que sean requisito de su concurrencia. Así ocurre con el involucramiento de fuerzas estatales u organizadas en la comisión de los crímenes; declaraciones, instrucciones o documentación atribuible al Estado o a la organización que consiente o promueve la comisión de los crímenes; y una motivación subyacente, entre otros ${ }^{39}$.

Asimismo, se ha sostenido también que la política no necesariamente ha de existir con anterioridad al ataque, pudiendo surgir conforme se van cometiendo los actos inhumanos ${ }^{40}$.

ii) El origen de la política

En cuanto al origen de la política, luego de una discusión inicial acerca del tema ${ }^{41}$ ha quedado establecido que no es necesario que la organización de la que ella emana tenga una estructura que presente las características formales de un Estado. Antes bien, se ha explicitado que la naturaleza formal del grupo y su nivel de organización no se consideran definitorios. En cambio, la organización debe disponer de medios suficientes, de estructuras o mecanismos suficientemente eficaces, de capacidad de acción y de concertación con el fin de asegurar la coordinación necesaria para llevar adelante el ataque, promoverlo o apoyarlo ${ }^{42}$.

35 CPI, 30.9.2008, N ICC-01/04-01/07, pfo. 396 ; CPI, 31.3.2010, No ICC-01/09, pfo. 84 ; 15.11.2011, No ICC-02/11, pfo. 43; CPI, 23.1.2012, No ICC-01/09-02/11, pfo. 111 ; CPI, 12.6.2014, $\mathrm{N}^{\circ}$ ICC-02/11-01/11, pfo. 215.

${ }^{36}$ CPI, 12.6.2014, N ICC-02/11-01/11, pfo. 210 ; CPI, 21.3.2016, No ICC-01/05-01/08, pfo. 160 ; CPI, 22.5.2018, No ICC-01/12-01/18, pfo. 49 ; CPI, 8.7.2019, No ICC-01/04-02/06, pfo. 674 ; CPI, 8.11.2019, No ICC-01/12-01/18, pfo. 143.

37 CPI, 30.9.2008, N ${ }^{\circ}$ ICC-01/04-01/07, pfo. 396 ; CPI, 31.3.2010, No ICC-01/09, pfo. 84 ;

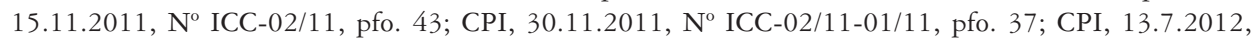
$\mathrm{N}^{\circ}$ ICC-01/04-02/06, pfo. 24.

${ }^{38}$ CPI, 21.3.2016, No ICC-01/05-01/08, pfo. 160 ; CPI, 22.5.2018, No ICC-01/12-01/18, pfo. 49 ;

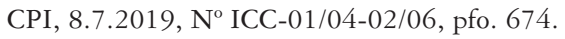

39 CPI, 21.3.2016, No ICC-01/05-01/08, pfo. 160 ; CPI, 8.7.2019, Nº ICC-01/04-02/06, pfo. 674. Más ejemplos de indicios de la existencia o implementación de una política se encuentran en CPI, 31.3.2010, No ICC-01/09, pfo. 87, con más referencias; CPI, 23.1.2012, No ICC-01/09-01/11, pfo. 219.

${ }^{40}$ CPI, 7.3. 2014, N ${ }^{\circ}$ ICC-01/04-01/07, pfo. 1110 ; CPI, 8.7.2019, No ICC-01/04-02/06, pfo. 674.

41 A partir de la opinión del juez Kaul en cuanto a que debía tratarse de entidades cuasiestatales. Pueden consultarse, por todos, Werle/Burghard, 2013, pp. 289 y ss.; Hall et al., 2016, pp. 247 y ss.

${ }^{42}$ CPI, 7.3. 2014, No ICC-01/04-01/07, pfo. 1119 y ss.; CPI, 21.3.2016, No ICC-01/05-01/08, pfo. 158; CPI, 9.11.2017, No ICC-01/17-X-9-US-Exp, 25 October 2017, pfo. 32; CPI, 22.5.2018, N ICC-01/1201/18, pfo. 47 ; CPI, 8.11.2019, No ICC-01/12-01/18, pfo. 156; CPI, 14.11.2019, N ICC-01/19, pfo. 63. 
Se ha explicitado que la determinación de si un grupo constituye una organización relevante ha de realizarse caso a caso. Para ello pueden considerarse, entre otros, los siguientes factores: (i) si el grupo tiene un mando responsable o una jerarquía establecida; (ii) si tiene de hecho los medios para llevar a cabo un ataque generalizado o sistemático contra una población civil; (iii) si ejerce control sobre parte del territorio del Estado; (iv) si tiene como propósito principal actividades criminales contra la población civil; (v) si articula, de manera implícita o explícita, una intención de atacar a una población civil; (vi) si es parte de un grupo mayor, que cumple algunos o todos los criterios anteriores. Por último, se insiste en que estos factores podrán asistir a la CPI en su determinación, pero no constituyen una definición legal rígida, que deba cumplirse exhaustivamente ${ }^{43}$.

En lo concerniente a la determinación de una política estatal, se ha establecido que una política estatal de cometer un ataque no necesariamente requiere ser concebida en los niveles más altos de la maquinaria del Estado. Una política adoptada por órganos regionales, locales o en general por parte del Estado, podría satisfacer el requisito de una política estatal ${ }^{44}$.

iii) La relación entre política y los actos del art. 7.1 ER

Se ha entendido que dentro de la estructura del delito, la política es lo que hace que los múltiples actos del art. 7.1 ER sean inteligibles como una línea de conducta en el sentido del 7.2 ER ${ }^{45}$. Una línea de conducta se opondría a un acto aislado. Ella describe algunos eventos, por oposición a una mera agregación de actos al azar ${ }^{46}$. Para demostrar que la línea de conducta fue llevada adelante en cumplimiento de la política, o para promoverla, ella debe reflejar un vínculo con la política, lo que permitiría excluir actos cometidos por individuos actuando por su cuenta, con independencia de ella. El requisito se satisface cuando se actúa deliberadamente para promover la política, o se lleva adelante deliberadamente actos acordes con ella. No se requiere en cambio que se compruebe que quienes actúan se han motivado por la política, o son miembros de la organización o el Estado del que ella emana ${ }^{47}$.

En el mismo sentido, CPI, 30.9.2008, No ICC-01/04-01/07, pfo. 396. Vid. asimismo CPI, 31.3.2010, $\mathrm{N}^{\circ}$ ICC-01/09, pfo. 90 y ss., con más referencias; CPI, 23.1.2012, No ICC-01/09-02/11, pfo. 112; CPI, 23.1.2012, No ICC-01/09-01/11, pfo. 184.

${ }^{43}$ CPI, 31.3.2010, N ICC-01/09, pfo. 93, con más referencias; CPI, 23.1.2012, No ICC-01/09-02/11, pfo. 109; CPI, 15.11.2011, No ICC-02/11, pfo. 46; CPI, 23.1.2012, No ICC-01/09-01/11, pfo. 184. Vid. Asimismo CPI, 12.6.2014, No ICC-02/11-01/11, pfo. 217.

${ }^{44}$ CPI, 31.3.2010, No ICC-01/09, pfo. 89, con más referencias; CPI, 15.11.2011, N ${ }^{\circ}$ ICC-02/11, pfo. 45; CPI, 23.1.2012, No ICC-01/09-02/11, pfo. 109; CPI, 12.6.2014, No ICC-02/11-01/11, pfo. 220.

${ }^{45}$ CPI, 7.3. 2014, N ICC-01/04-01/07, pfo. 1114; CPI, 22.5.2018, No ICC-01/12-01/18, pfo. 50.

46 CPI, 12.6.2014, No ICC-02/11-01/11, pfo. 209; CPI, 21.3.2016, No ICC-01/05-01/08, pfo. 149; CPI, 9.11.2017, No ICC-01/17-X-9-US-Exp, 25 October 2017, pfo. 32; CPI, 14.11.2019, N ICC-01/19,

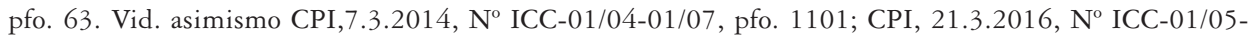
01/08, pfo. 149.

${ }^{47}$ CPI, 21.3.2016, N ICC-01/05-01/08, pfo. 161, con más referencias; CPI, 9.11.2017, N ICC01/17-X-9-US-Exp, 25 October 2017, pfo. 32; CPI, 22.5.2018, No ICC-01/12-01/18, pfo. 50 ; CPI, 14.11.2019, No ICC-01/19, pfo. 63. 


\section{b) Situaciones y casos concretos}

En lo que sigue se listan ejemplos concretos de decisiones relevantes respecto de la política en los crímenes de lesa humanidad.

- En la situación de Kenia, para ejecutar el plan, se habría creado una red de personas poderosas o influyentes de la política, los medios de comunicación masiva, la economía, la policía y la milicia; y se habrían distribuido recursos y planificado cómo se actuaría, creando una estructura con distintos niveles de mando y áreas de competencia, además de modus operandi definidos. Para ganar adeptos, se habría adoctrinado a la población diciendo que la elección estaría arreglada y que Kibaki les perseguiría luego de ella ${ }^{48}$. En la misma situación, se explicitó que, si bien una política no necesariamente equivale a un plan, en los hechos ambos pueden superponerse $^{49}$. Para considerar que los Mungiki calificarían como una organización en el sentido del art. 7.2.a) ER fue relevante su organización jerárquica, bajo el control de una persona; la existencia de un sistema efectivo de asegurar el cumplimiento de las reglas y órdenes impuestos por los más altos niveles de mando; el gran tamaño de la organización, que incluía un ala cuasimilitar; y que en ciertos lugares de Kenia habría proveído de servicios sociales y seguridad ${ }^{50}$.

- En la situación de RDC, en el caso Mbarushimana, se consideró que la política podría consistir en crear una catástrofe humanitaria, de modo que el gobierno y líderes extranjeros dejen de molestar a las denominadas "Fuerzas Democráticas para la Liberación de Ruanda" 51 . En definitiva, en ese caso, tal política no se probó a satisfacción de la Sala. Al efecto, se consideraron relevantes -entre otros factores- declaraciones de testigos, además de textos y declaraciones públicas de la organización ${ }^{52}$. Asimismo, en el caso Ntaganda se consideró que la Unión de Patriotas Congoleños y las Fuerzas Patrióticas para la Liberación del Congo habrían contado con los medios para realizar un ataque contra una población civil, pues habrían tenido acceso a una cantidad relevante de armas, a personal con entrenamiento militar y a líneas de comunicación ${ }^{53}$.

- En la situación de Libia se consideró relevante una política diseñada al más alto nivel del aparato estatal, "encaminada a impedir y sofocar, por cualquier medio -incluido el uso de la fuerza letal-, las manifestaciones de civiles” contra el

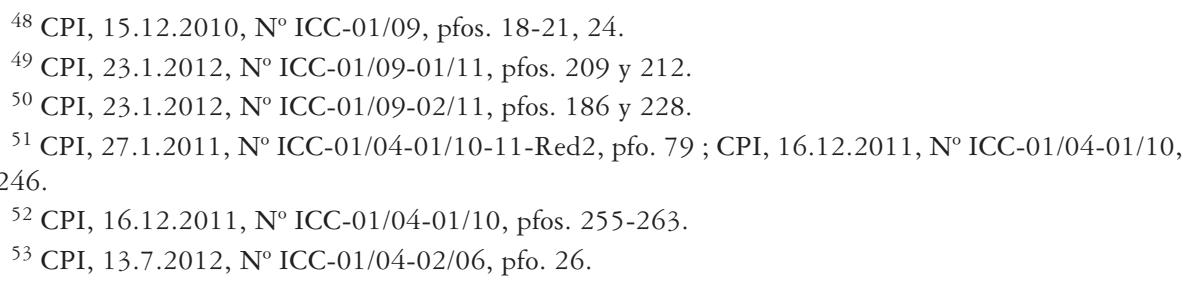


gobierno ${ }^{54}$. Se consideró la evidencia de que se habría ordenado a las "Fuerzas de Seguridad" disciplinar a los manifestantes; de que dichas "Fuerzas de Seguridad" se encontraban desplegadas por todo el país; y de que se habrían tomado medidas para reforzarlas, de cara a las manifestaciones. También se consideró relevante la información acerca de una campaña tendiente a evitar que se informara respecto de ciertos hechos y castigar a quienes lo hicieran ${ }^{55}$.

- En la situación de Costa de Marfil se consideró que el círculo íntimo del expresidente Gbagbo y las fuerzas pro-Gbabo habrían conformado una organización para los efectos del art. 7.2.a) ER, y que habrían adoptado una política de llevar adelante ataques violentos contra sus oponentes en la elección presidencial de 2010, buscando retener el poder por todos los medios ${ }^{56}$. Asimismo, se consideró que la política debe haber sido implementada por la conducta del Estado o de la organización ${ }^{57}$. En la misma situación se consideró que la naturaleza de los actos, lo que se perseguía con ellos, la población que fue objeto del ataque y la cronología y lugares de los eventos relevantes hacen que esos múltiples actos, apreciados colectivamente, expresen una línea de conducta y se consideren cometidos como parte del ataque, en el sentido del art. $7.1 \mathrm{ER}^{58}$.

- En la situación de Burundi se consideró que existió una política estatal consistente en suprimir visiones disidentes y opositoras al tercer periodo presidencial del presidente Nkurunziza ${ }^{59}$.

- En un caso de la situación concerniente a Mali se determinó que los grupos armados organizados, en el sentido del derecho internacional humanitario, cumplen con las características de una organización de la que puede emanar una política relevante para crímenes de lesa humanidad ${ }^{60}$, y que tal política estaría definida, en Mali, por la voluntad de los grupos armados de imponerse a la población, lo mismo que imponer a la población el orden religioso que pretendían favorecer. Para ello, se habría seguido un patrón regular consistente en aplicar reglas, prohibiciones y castigos, dirigidas con particular estrictez a mujeres y niñas ${ }^{61}$. En Mali, el ataque contra una población civil se dio en un ambiente en el que -a pretexto de mantener el orden público- la policía islámica podía infligir castigos considerados menores, sin intervención de un tribunal, para reprimir comportamientos que

${ }^{54}$ CPI, 27.6.2011, No ICC-01/04-02/06, pfo. 31 ; CPI, 27.6.2011, No ICC-01/11, p. 4; CPI, 18.4.2013, $\mathrm{N}^{\circ}$ ICC-01/11-01/13, pfo. 5 y ss.

${ }^{55}$ CPI, 27.6.2011, No ICC-01/04-02/06, pfo. 28-30.

56 CPI, 16.11.2011, No ICC-02/11, pfo 5 ; CPI, 30.11.2011, No ICC-02/11-01/11, pfos. 46-47; CPI, 29.2.2012, $\mathrm{N}^{\circ}$ ICC-02/11-01/12, pfo. 6; CPI, 2.3.2012, $\mathrm{N}^{\circ}$ ICC-02/11-01/12, pfos. 20 y 21 ; CPI,

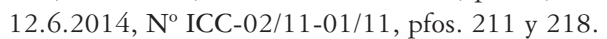

${ }^{57}$ CPI, 30.11.2011, No ICC-02/11-01/11, pfo. 37.

${ }^{58} \mathrm{CPI}, 12.6 .2014, \mathrm{~N}^{\circ}$ ICC-02/11-01/11, pfo. 212.

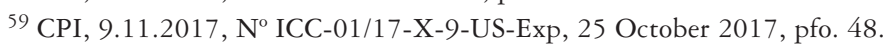

${ }^{60}$ CPI, 22.5.2018, No ICC-01/12-01/18, pfo. 59 ; CPI, 8.11.2019, No ${ }^{\circ}$ ICC-01/12-01/18, pfo. 172.

${ }^{61}$ CPI, 22.5.2018, No ICC-01/12-01/18, pfo. 60. 
considerara desviados, y prevenir su repetición. Allí la cronología de los eventos, la toma de un poblado por la fuerza, la creación de nuevos órganos, los distintos aspectos de la política de los grupos y su implementación sistemática mostrarían la existencia de un vínculo entre los distintos actos que hacen que se trate de una línea de conducta ${ }^{62}$.

\section{El Carácter generalizado o Sistemático del ataque}

El requisito de que se trate de un ataque generalizado o sistemático es consuetudinario y estatutario (art. 7.1 ER), y se reafirma en los EC para el art. 7, introducción, pfo. 3.

\section{Criterios abstractos en las resoluciones de la Corte Penal Internacional}

Existe consenso en que con este requisito disyuntivo se busca excluir actos cometidos aisladamente o hechos ocurridos al azar de la noción de crímenes de lesa humanidad ${ }^{63}$.

\section{a) Ataque generalizado}

Se ha entendido que el término "generalizado" caracteriza un ataque a gran escala, que está dirigido contra un gran número de personas ${ }^{64}$. Puede tratarse de un ataque llevado adelante en un área geográfica extendida o en un área geográfica pequeña contra un gran número de civiles ${ }^{65}$. Un ataque podría ser generalizado ya sea por el efecto acumulativo de varios actos inhumanos o por el efecto de un acto inhumano singular de extraordinaria magnitud ${ }^{66}$. En particular desde 2010 se viene poniendo de relieve que para la caracterización de un ataque como generalizado deben considerarse todos

${ }^{62}$ CPI, 8.11.2019, Nº ICC-01/12-01/18, pfo. 344. La descripción de los hechos del ataque se encuentra entre los pfos. $175,269-329$ y $339-349$.

${ }^{63}$ CPI, 30.9.2008, Confirmation charges Katanga/Ngudjolo Chui, pfo. 394, con más referencias; CPI, 31.3.2010, Authorization investigation Kenya, pfo. 94, con más referencias; CPI, 23.1.2012, Confirmation charges Muthaura/Kenyatta/Ali, pfo. 109.

${ }^{64}$ CPI, 30.9.2008, No ICC-01/04-01/07, pfo. 394, con más referencias; CPI, 15.7.2009, N ${ }^{\circ}$ ICC01/05-01/08, pfo. 83; CPI, 23.1.2012, No ICC-01/09-02/11, pfo. 109; CPI, 1.3.2012, No ICC-02/05-01/12, pfo. 62; CPI,7.3.2014, N ${ }^{\circ}$ ICC-01/04-01/07, pfo. 1123; CPI, 15.11.2011, No ICC-02/11, pfo. 53, con más referencias; CPI, 21.3.2016, No ICC-01/05-01/08, pfo. 163; CPI, 12.6.2014, No ICC-02/11-01/11, pfo. 222; CPI, 9.11.2017, N ICC-01/17-X-9-US-Exp, 25 October 2017, pfo. 32; CPI, 8.7.2019, N ICC01/04-02/06, pfo. 691; CPI, 14.11.2019, N ICC-01/19, pfo. 63.

${ }^{65}$ CPI, 30.9.2008, No ICC-01/04-01/07, pfo. 395; CPI, 15.7.2009, No ICC-01/05-01/08, pfo. 83, con más antecedentes; CPI, 15.11.2011, No ICC-02/11, pfo. 53, con más antecedentes; CPI, 23.1.2012, $\mathrm{N}^{\circ}$ ICC-01/09-02/11, pfo. 109.

${ }^{66}$ CPI, 31.3.2010, No ICC-01/09, pfo. 95, con más referencias; CPI, 30.11.2011, No ICC-02/11-01/11, pfo. 49; CPI, 23.1.2012, No ICC-01/09-02/11, pfo. 109; CPI, 1.3.2012, No ICC-02/05-01/12, pfo. 51. 
los hechos relevantes del caso, no exclusivamente datos cuantitativos o geográficos ${ }^{67}$. La duración temporal del ataque no tendría, en todo caso, impacto en esta parte del análisis ${ }^{68}$.

Las características de "masivo, frecuente, llevado adelante con seriedad considerable y dirigido contra multiplicidad de víctimas" serían necesarias, de acuerdo con un grupo de resoluciones, para que el ataque sea generalizado ${ }^{69}$, mientras que en otras resoluciones, en general más recientes, se considera que estas características pueden estar presentes como factores relevantes a esa determinación, sin que se trate sin embargo de requisitos ${ }^{70}$.

\section{b) Ataque sistemático}

Existe consenso en las resoluciones respecto de que el término "sistemático" refleja la naturaleza organizada de los actos de violencia, y la improbabilidad de su ocurrencia al $\operatorname{azar}^{71}$. Esta caracterización a menudo se expresa en la existencia de patrones criminales, evidenciados por la repetición no accidental, de conducta criminal similar, con regularidad $^{72}$. Se ha entendido que el ataque es sistemático si se puede establecer que existe un modus operandi, o si reiteradamente se lleva adelante la misma clase de conductas

${ }^{67}$ CPI, 31.3.2010, N ICC-01/09, pfo. 95; CPI, 15.11.2011, No ICC-02/11, pfo. 53; CPI, 23.1.2012,

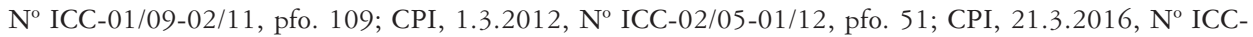
01/05-01/08, pfo. 163; CPI, 12.6.2014, N ICC-02/11-01/11, pfo. 222; CPI, 8.7.2019, No ICC-01/0402/06, pfo. 691.

${ }^{68}$ CPI, 21.3.2016, No ICC-01/05-01/08, pfo. 163; CPI, 9.11.2017, No ICC-01/17-X-9-US-Exp, 25 October 2017, pfo. 32; CPI, 14.11.2019, No ICC-01/19, pfo. 63.

${ }^{69}$ CPI, 6.7.2007, No ICC-01/04-01/07, pfo. 33 ; CPI, 6.7.2007, No ICC-01/04-02/07, pfo. 34 ;

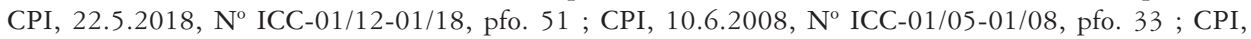
15.7.2009, No ICC-01/05-01/08, pfo. 83; CPI, 31.3.2010, No ICC-01/09, pfo. 95, con más referencias;

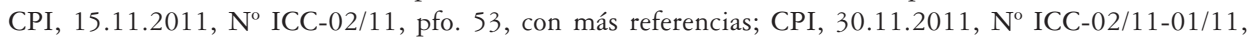
pfo. 49; CPI, 23.1.2012, N ICC-01/09-02/11, pfo. 109; CPI, 13.7.2012, No ICC-01/04-02/06, pfo. 9 ; CPI, 12.6.2014, No ICC-02/11-01/11, pfo. 222. En el caso contra Ruto y Sang se estimó que esas características concurrían, lo que haría que hubiera motivos fundados para creer que el ataque había sido generalizado CPI,

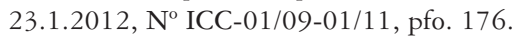

${ }^{70}$ CPI, 8.7.2019, No ICC-01/04-02/06, pfo. 691; CPI, 21.3.2016, No ICC-01/05-01/08, pfo. 163; CPI, 9.11.2017, N ICC-01/17-X-9-US-Exp, 25 October 2017, pfo. 32; CPI, 14.11.2019, No ICC-01/19, pfo. 63.

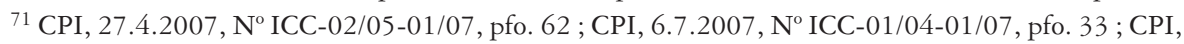

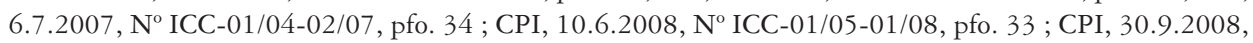
$\mathrm{N}^{\circ}$ ICC-01/04-01/07, pfo. 397; CPI, 31.3.2010, $\mathrm{N}^{\circ}$ ICC-01/09; pfo. 96; CPI ; 27.1.2011, N ICC-01/0401/10-11-Red2, pfo. 91 ; CPI, 15.11.2011, No ICC-02/11, pfo. 54, con más referencias; CPI, 30.11.2011, $\mathrm{N}^{\circ}$ ICC-02/11-01/11, pfo. 49; CPI, 23.1.2012, $\mathrm{N}^{\circ}$ ICC-01/09-01/11, pfo. 179; CPI, 13.7.2012, No ICC01/04-02/06, pfo. 9 ; CPI, 7.3.2014, No ICC-01/04-01/07, pfos. 1098, 1111 у 1123; CPI, 12.6.2014, $\mathrm{N}^{\circ}$ ICC-02/11-01/11, pfos. 209, 223; CPI, 9.11.2017, No ICC-01/17-X-9-US-Exp, 25 October 2017, pfo. 32; CPI, 8.7.2019, N ICC-01/04-02/06, pfo. 692; CPI, 14.11.2019, No ICC-01/19, pfo. 63.

${ }^{72}$ CPI, 30.9.2008, No ICC-01/04-01/07, pfo. 397; CPI, 31.3.2010, No ICC-01/09; pfo. 96, con más referencias; CPI, 15.11.2011, N $\mathrm{N}^{\circ}$ ICC-02/11, pfo. 45, con más antecedentes; CPI, 30.11.2011, N $\mathrm{N}^{\circ}$ ICC02/11-01/11, pfo. 49; CPI, 13.7.2012, N ICC-01/04-02/06, pfo. 31 ; CPI, 7.3.2014, No ICC-01/04-01/07, pfo. 1123; CPI, 12.6.2014, No ICC-02/11-01/11, pfos. 209 y 223; CPI, 9.11.2017, No ICC-01/17-X-9-USExp, 25 October 2017, pfo. 32; CPI, 8.7.2019, $\mathrm{N}^{\circ}$ ICC-01/04-02/06, pfo. 692; CPI, 8.11.2019, N ${ }^{\circ}$ ICC-

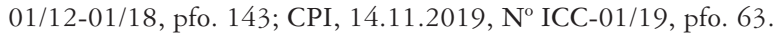


tendientes a la misma clase de efectos en la población civil objeto del ataque ${ }^{73}$. También se ha señalado que la existencia de una política del Estado o de la organización es un elemento a partir del cual se puede inferir el carácter sistemático del ataque ${ }^{74}$.

\section{Situaciones y casos concretos}

- En la situación de Darfur (Sudán), se entendió que el ataque era generalizado, pues habría afectado, por lo menos, a centenares de miles de personas y habría tenido lugar en grandes porciones del territorio de la región de Darfur; siendo además de sistemático, pues los actos de violencia de que se trata se habrían inscrito, en gran medida, en una serie de actos análogos ${ }^{75}$, que habrían seguido un patrón similar, extendiéndose por más de cinco años ${ }^{76}$. Además, se consideró relevante que se habría seguido una estrategia unificada a nivel nacional, con una estructura para organizar el quehacer de las Fuerzas Armadas sudanesas, la policía y los demás grupos encargados de ejecutar el ataque ${ }^{77}$.

- Un ataque relevante puede tener, a saber, una duración de dos meses (Kenia) ${ }^{78}$, algo más de seis meses (Libia) ${ }^{79}$ u 8 meses (Mali) ${ }^{80}$. Se considera que los crímenes se han cometido por un periodo extendido de tiempo (indicio de un ataque generalizado y sistemático) cuando este comprendió entre abril de 2012 y enero de $2013^{81}$.

- Se considera que el área geográfica comprendida por dos distritos de una provincia de Kenia, constituyen un área geográfica amplia. Se atiende además al número de personas que han sido sujetos pasivos de delitos (más de 237 muertes, más de 505 heridos, más de 500 personas desplazadas, viviendas y negocios saqueados y quemados) para establecer el carácter generalizado del ataque ${ }^{82}$.

- En la sentencia del caso Katanga (RDC), la Sala de Primera Instancia (SPI) II ha dicho que el análisis del carácter sistemático de un ataque va más allá de la existencia de una política. Se explicitó que para caracterizar un ataque como sistemático se requeriría probar la existencia de un patrón de conducta o la existencia de una comisión continua o recurrente de hechos vinculados entre sí, que no sería necesario probar para dar por establecida la sola existencia de una política, que

${ }^{73}$ CPI, 7.3.2014, N ${ }^{\circ}$ ICC-01/04-01/07, pfo. 1113; CPI, 8.7.2019, N ${ }^{o}$ ICC-01/04-02/06, pfos. 693, 695.

${ }^{74}$ CPI, 27.4.2007, N ${ }^{\circ}$ ICC-02/05-01/07. pfo. 62; CPI, 10.6.2008, N ${ }^{\circ}$ ICC-01/05-01/08, pfo. 33; CPI, 13.7.2012, No ICC-01/04-02/06, pfo. 9; CPI, 31.3.2010, Nº ICC-01/09, pfo. 96, con más referencias.

${ }^{75}$ CPI, 4.3.2009, N ${ }^{\circ}$ ICC-02/05-01/09 (arrest warrant), p. 5.

76 CPI, 4.3.2009, $\mathrm{N}^{\circ}$ ICC-02/05-01/09 (art. 58), pfo. 85.

77 CPI, 27.4.2007, N ${ }^{\circ}$ ICC-02/05-01/07. pfo. 65-66.

${ }^{78}$ CPI, 31.3.2010, No ICC-01/09; pfo. 131.

${ }^{79}$ CPI, 18.4.2013, N ${ }^{\circ}$ ICC-01/11-01/13, pfo. 5.

${ }^{80}$ CPI, 8.11.2019, No ICC-01/12-01/18, pfo. 344.

${ }^{81}$ CPI, 27.3.2018, No ICC-01/12-01/18, pfo. 11.

${ }^{82}$ CPI, 23.1.2012, N ${ }^{\circ}$ ICC-01/12-01/18, pfos. 177, 178. 
por su parte requeriría solamente probar que el Estado o la organización tenían la voluntad de que se cometiera un ataque contra una población civil ${ }^{83}$.

- En la situación de Costa de Marfil se consideró que el ataque tuvo una gran escala y fue por lo mismo generalizado de acuerdo con el art. 7.1 ER, considerando el efecto acumulativo de varios actos de violencia, porque involucró un gran número de actos; apuntó y victimizó a un número significativo de individuos; se extendió por un periodo de más de cuatro meses; y afectó a toda una ciudad de más de tres millones de habitantes. Además, el ataque habría sido también sistemático porque habría evidencia de preparativos y se habría tratado de un ataque coordinado y planificado, obedeciéndose además patrones de violencia ${ }^{84}$.

- En la situación de Mali se estimó que el carácter generalizado y sistemático del ataque se demostró, entre otros factores, por el periodo extendido en el que se cometieron los crímenes, el alto número de víctimas, los medios empleados para cometer los crímenes, las estructuras establecidas en fomento de su comisión, y la existencia de un patrón general en el modo de cometerlos ${ }^{85}$. Para valorar el carácter sistemático del ataque, se consideró que la promulgación de prohibiciones fue sistemática, y que habría existido un modus operandi violento, además de una organización estructurada para llevar a cabo el ataque ${ }^{86}$.

\section{RESPECTO DE LOS HALlAZGOS}

La principal novedad del ER en cuanto a la descripción del ataque contra una población civil es que cuenta con una definición de ataque en el art. 7.2 ER, que incluye a una política como uno de sus elementos ${ }^{87}$. Hasta entonces, el plan o política se consideraban indiciarios del carácter sistemático del ataque ${ }^{88}$. Está vastamente documentado que la inclusión del 7.2 ER se decidió en las negociaciones, a cambio de mantener en el enunciado principal del art. 7.1 ER la tradicional conjunción disyuntiva "o", que exige solo una de las características (generalizado o sistemático) del ataque para que este sea típico ${ }^{89}$.

A partir de lo reseñado en los apartados anteriores puede afirmarse que no se ha superado el problema, identificado a más tardar a partir de la resolución que denegó confirmar los cargos en el caso Mbarushimana, de que existe una tendencia importante

${ }^{83}$ CPI, 7.3.2014, N ${ }^{\circ}$ ICC-01/04-01/07, pfo. 1113.

${ }^{84}$ CPI, 12.6.2014, No ICC-02/11-01/11, pfos. 224 y 225. Vid también CPI, 23.11.2011, N ${ }^{\circ}$ ICC-

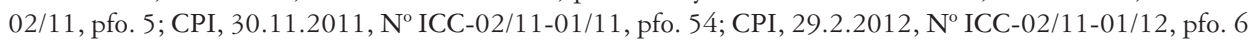
y CPI, 15.11.2011, No ICC-02/11, pfo. 62.

${ }^{85}$ CPI, 27.3.2018, N ${ }^{\circ}$ ICC-01/12-01/18, pfo. 11.

${ }^{86}$ CPI, 22.5.2018, No ICC-01/12-01/18, pfos. 63-65.

${ }^{87}$ Con más antecedentes, HALL et al., 2016, 244.

88 TPIY, 12.6.2002, IT-96-23\&IT-96-23/1-A, pfo. 98.

${ }^{89}$ Con más antecedentes, WERLE y JESSBERGER, 2017, p. 566. 
dentro de las resoluciones de la CPI en cuanto a confundir lo que ha de comprobarse para satisfacer el elemento político con lo que ha de satisfacerse para entender que el ataque ha tenido carácter sistemático. Eso acarrea problemas tanto en lo concerniente a la interpretación sistemática de las disposiciones del ER como en lo relativo a una sobreexigencia en cuanto a la tipicidad de la conducta, al exigirse, en la práctica, la sistematicidad del ataque en todos los casos, cuando en el tipo esa característica se ha tratado invariablemente como una alternativa al carácter generalizado del ataque ${ }^{90}$. Lo anterior puede aseverarse pues, a pesar de los esfuerzos en distinguir, los hechos y las consideraciones en las que se basan las decisiones coinciden respecto del elemento político y la característica de sistematicidad. Estos esfuerzos en distinguir han existido tanto por parte de la doctrina ${ }^{91}$ como por parte de la CPI. En las resoluciones de la CPI, si se compara las primeras con las más recientes, se puede constatar que en ellas se ha llegado a estandarizar una estructura de contenidos, en la que hoy se suele distinguir -incluso con epígrafes distintos- entre la política del Estado o de la organización que engarza a esos actos como una línea de conducta (incluyendo allí la identificación de la población civil atacada) y el carácter sistemático o generalizado del ataque. Con todo, lo que se examina en ambos apartados suele no ser distinguible, a pesar de intentos de explicación ${ }^{92}$. De hecho -como se ha reseñado supra- se ha seguido utilizando el criterio (asentado desde los tribunales ad hoc) de acuerdo al cual el hecho de que se haya seguido un plan o política constituye un indicio de la sistematicidad del ataque ${ }^{93}$, o se ha dicho que, si bien una política no necesariamente equivale a un plan, en los hechos ambos pueden superponerse $^{94}$, o que ambos se refieren a un cierto nivel de planificación del ataque ${ }^{95}$.

Acaso tenga que llegar a conocimiento de las Salas algún caso de crímenes de lesa humanidad en los que no se considere existente el carácter sistemático del ataque, pero sí la política, para poder apreciar con nitidez cómo esa diferencia queda reflejada en la práctica. Por ahora, eso parece ser un empeño que ha resultado mejor en la doctrina y en la estructura formal que en el fondo de las resoluciones de la CPI.

\section{CONCLuSiones}

Tanto en el ER como en los EC se encuentran descripciones de lo que se entiende por ataque contra una población civil, sus elementos y características. Para el ejercicio de su interpretación es correcto valerse de las resoluciones de la CPI, como medio de determinación de reglas, junto con la doctrina. En este empeño, no debe olvidarse que la CPI fue creada para ejercer jurisdicción sobre los crímenes más graves de trascendencia

\footnotetext{
${ }^{90}$ Sobre eso, a saber, ROBINSON 2015, pp. 705 y ss.

91 A saber, ROBINSON 2015, pp. 705 y ss., en especial 714.

92 CPI, 7.3.2014, N ICC-01/04-01/07, pfo. 1113; CPI, 12.6.2014, No ICC-02/11-01/11, pfo. 216.

93 Tal criterio es resaltado por SAFFERLING, 2011, p. 193, con más referencias.

${ }^{94}$ CPI, 23.1.2012, No ICC-01/09-01/11, pfos. 209 y 212.

${ }^{95}$ CPI, 12.6.2014, N ${ }^{\circ}$ ICC-02/11-01/11, pfo. 216.
} 
internacional, en situaciones de excepción, y no para conocer de todas las conductas punibles de acuerdo con el derecho internacional. Por eso, lo que resulta de la revisión de resoluciones que se ha presentado no tiende a determinar estándares mínimos para la punibilidad de las conductas, sino - antes bien- a mostrar lo decidido respecto de los más graves crímenes contra el derecho internacional.

Algunos criterios generales de interpretación han mostrado constancia en el tiempo, de modo que incluso las mismas Salas de la CPI los califican como establecidos (established case law of the Court $)^{96}$. Así ocurre, a saber, con los siguientes:

- El entendimiento del ataque como una campaña u operación dirigida contra una población civil, que puede estar constituido por cualquier forma de violencia contra ella.

- La comprensión de que la multiplicidad de actos del art. 7.1 ER que lo constituyen han de ser más de "algunos" o "varios", sin que esto imponga una cantidad mínima para cada forma de cometer crímenes de lesa humanidad.

- La comprensión de una población como un colectivo que se distingue del resto de las personas por algún factor cualquiera, y que tiene que ser ella el objeto no incidental del ataque.

- La aplicación de las normas del derecho internacional humanitario para determinar el carácter civil de una población en caso de conflicto armado.

- La afirmación de que la política no necesariamente debe ser explícita, sino que su existencia puede ser inferida de una serie de factores, y que ella puede emanar de cualquier organización que disponga de la capacidad necesaria para llevar adelante un ataque, promoverlo o apoyarlo.

- El entendimiento de que, para ser parte de una línea de conducta, los actos han de llevarse adelante deliberadamente de acuerdo con la política, sin que sea necesario que hayan sido motivados por ella.

- La necesidad de considerar todos los hechos para valorar un ataque como generalizado, sin limitarse necesariamente a datos cuantitativos o geográficos, que sin embargo suelen ser suficientes para dar cuenta de la escala de la situación.

- La determinación de que un factor relevante al momento de valorar un ataque como sistemático es la repetición de conductas criminales que guarden ciertas similitudes entre sí, con una regularidad que haga improbable su atribución al azar.

En las situaciones y casos, puede notarse que los hallazgos siguen la línea de los criterios generales recién reseñados.

Resulta destacable que si bien hay esfuerzos por diferenciar el elemento político del carácter sistemático del ataque a la hora de abordar su análisis -en el sentido de expresar que el primero es menos exigente que el segundo- no acaba este de ser un

${ }^{96}$ También "The established jurisprudence of the Court". CPI, 23.1.2012, No ICC-01/09-02/11, pfo. 109; CPI, 12.6.2014, No ICC-02/11-01/11, pfo. 215 ; CPI, 9.11.2017, No ICC-01/17-X-9-US-Exp, 25 October 2017, pfo. 32; CPI, 14.11,2019, N ICC-01/19, pfo. 63. 
asunto plenamente logrado en el conjunto de resoluciones, ni tampoco en todas las más recientes. Esto puede deberse en parte a que los factores que se enumeran como relevantes para inferir a partir de ellos la existencia de una política coinciden vastamente con características que, de existir, hacen que el ataque pueda entenderse como uno sistemático. Seguramente será este uno de los asuntos que -junto con otros- se desarrollarán más en resoluciones posteriores de esta todavía joven jurisdicción penal internacional permanente.

\section{BiBLIOGRAFÍA}

Alija Fernández, Rosa Ana y Saura Estapà, Jaume, 2016: "Towards a Single and Comprehensive Notion of 'Civilian Population' in Crimes against Humanity”, International Criminal Law Review vol. 16, pp. 1-31.

Beltrán Carpentier, Pablo, 2013: "Requisitos comunes a todo crimen de lesa humanidad". En: Claudia Cárdenas Aravena y Karinna Fernández Neira (editoras), La Corte Penal Internacional y sus primeros 10 años: un enfoque práctico, Santiago, Thomson Reuters, Santiago, pp. 49-59.

Eboe-Osuji, Chile, 2008: "Crimes Against Humanity: Directing Attacks Against A Civilian Population”, African Journal of Legal Studies, pp. 118-129.

Hall, Christopher Keith et al., 2016: “Article 7”. En: Kai Ambos y Otto Triffterer (editores), Commentary on the Rome Statute of the International Criminal Court, 3ra edición, Munich, Beck-Hart-Nomos, pp. 144-294.

Naciones Unidas, Texto del proyecto de artículos sobre los crímenes de lesa humanidad aprobado por la Comisión en primera lectura, disponible en UN Doc. A/72/10 > https://legal.un.org/ ilc/reports/2017/spanish/chp4.pdf<.

Rastan, Rod, 2013: “Situación y caso, definiendo los parámetros”. En: Claudia Cárdenas Aravena y Karinna Fernández Neira (editoras), La Corte Penal Internacional y sus primeros 10 años: un enfoque práctico, Santiago, Thomson Reuters, Santiago, pp. 209-251.

Robinson, Darryl, 2015: "Crimes against Humanity. A Better Policy on 'Policy”, en Carsten Stahn (editor), The Law and Practice of the International Criminal Court, Oxford, Oxford University Press, pp. 705-731.

SAdat, Leila Nadya, 2017, "Putting Peacetime First: Crimes against Humanity and the Civilian Population Requirement", Emory International Law Review vol. 31, pp. 197-269.

VV.AA. Journal of International Criminal Justice, Volume 16, Issue 4, September 2018, Special Issue: Laying the Foundations for a Convention on Crimes Against Humanity.

SAFFERLING, Christoph, 2011: Internationales Strafrecht, Heidelberg: Springer.

Werle, Gerhard y Burghardt, Boris, 2013, “¿Requieren los crímenes de lesa humanidad de la participación de un Estado o de una organización “cuasiestatal'?”. En: Claudia Cárdenas Aravena y Karinna Fernández Neira (editoras), La Corte Penal Internacional y sus primeros 10 años: un enfoque práctico, Santiago, Thomson Reuters, Santiago, pp. 289-309.

Werle, Gerhard y Jessberger, Florian, 2017, Tratado de Derecho Penal Internacional (3ra ed.), Valencia, Tirant lo Blanch.

Fuentes jurídicas y resoluciones judiciales citadas

Decision on the Prosecutions Application under article 58(7) of the Statute, de fecha 27 de abril de 2007, N ICC-02/05-01/07. 
CPI, SCP I, Decision on the evidence and information provided by the Prosecution for the issuance of a warrant of arrest for Germain Katanga, de fecha 6 de julio de 2007, No ICC-01/04-01/07.

CPI, SCP I, Decision on the evidence and information provided by the Prosecution for the issuance of a warrant of arrest for Mathieu Ngudjolo Chui, de fecha 6 de julio de 2007, $\mathrm{N}^{\circ}$ ICC-01/04-02/07.

CPI, SCP III, Decision on the Prosecutor's Application for a Warrant of Arrest against Jean-Pierre Bemba Gombo, de fecha 10 de junio de 2008, N ICC-01/05-01/08.

CPI, SCP I, Decision on the confirmation of charges, de fecha 30 de septiembre de 2008, $\mathrm{N}^{\circ}$ ICC-01/04-01/07.

CPI, SCP I, Public Redacted Version Decision on the Prosecution's Application for a Warrant of Arrest against Omar Hassan Ahmad Al Bashir, de fecha 4 de marzo de 2009, No ICC-02/05-01/09.

CPI, SCP I, Orden de detención de Omar Hassan Ahmad Al Bashir, de fecha 4 de marzo de 2009, No ICC-02/05-01/09.

CPI, SCP II, Decision Pursuant to Article 61(7)(a) and (b) of the Rome Statute on the Charges of the Prosecutor Against Jean-Pierre Bemba Gombo, de fecha 15 de julio de 2009, $\mathrm{N}^{\circ}$ ICC-01/05-01/08.

CPI, SCP II, Decision Pursuant to Article 15 of the Rome Statute on the Authorization of an Investigation into the Situation in the Republic of Kenya, de fecha 31 de marzo de 2010, $\mathrm{N}^{\circ}$ ICC-01/09.

CPI, SCP II, Prosecutor's Application Pursuant to Article 58 as to William Samoei Ruto, Henry Kiprono Kosgey and Joshua Arap Sang, de fecha 15 de diciembre de 2010, No ICC-01/09.

CPI, SCP I, Public Redacted Version Prosecution's Application under Article 58, de fecha 27 de enero de 2011, No ICC-01/04-01/10-11-Red2.

CPI, SCP II, Public redacted version Decision on the Prosecutor's Application under Article 58, de fecha 13 de junio de 2011, No ICC-01/04-02/06.

CPI, SCP I, orden de detención de Saif Al-Islam Qadhafi, de fecha 27 de junio de 2011, $\mathrm{N}^{\circ}$ ICC-01/11.

CPI, SCP III, Corrigendum to "Decision Pursuant to Article 15 of the Rome Statute on the Authorisation of an Investigation into the Situation in the Republic of Côte d'Ivoire", de fecha 15 de noviembre de 2011, No ICC-02/11.

CPI, SCP III, Orden de detención de Laurent Koudou Gbagbo, de fecha 23 de noviembre de

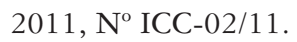

CPI, SCP III, Decision on the Prosecutor's Application Pursuant to Article 58 for a warrant of arrest against Laurent Koudou Gbagbo, de fecha 30 de noviembre de 2011, No ICC-02/11-01/11.

CPI, SCP I, Decision on the confirmation of charges, de fecha 16 de diciembre de 2011, $\mathrm{N}^{\circ}$ ICC-01/04-01/10.

CPI, SCP II, Decision on the Confirmation of Charges Pursuant to Article 61(7)(a) and (b) of the Rome Statute, de fecha 23 de enero de 2012, No ICC-01/09-02/11.

CPI, SCP II, Decision on the Confirmation of Charges Pursuant to Article 61(7)(a) and (b) of the Rome Statute, de fecha 23 de enero de 2012, No ICC-01/09-01/11.

CPI, SCP III, Warrant of Arrest for Simone Gbagbo, de fecha 29 de febrero de 2012, $\mathrm{N}^{\circ}$ ICC-02/11-01/12.

CPI, SCP I, Public redacted version of "Decision on the Prosecutor's application under article 58 relating to Abdel Raheem Muhammad Hussein”, de fecha 1 de marzo de 2012, $\mathrm{N}^{\circ}$ ICC-02/05-01/12.

CPI, SCP III, Decision on the Prosecutor's Application Pursuant to Article 58 for a warrant of arrest against Simone Gbagbo, de fecha 2 de marzo de 2012, No ICC-02/11-01/12. 
CPI, SCP II, Public redacted version Decision on the Prosecutor's Application under Article 58, de fecha 13 de julio de 2012, No ICC-01/04-02/06.

CPI, SCP II, Warrant of Arrest for Al-Tuhamy Mohamed Khaled, de fecha 18 de abril de 2013, $\mathrm{N}^{\circ}$ ICC-01/11-01/13.

CPI, SCP II, Judgment pursuant to article 74 of the Statute Katanga, de fecha 7 de marzo de

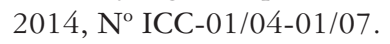

CPI, SCP I, Decision on the confirmation of charges against Laurent Gbagbo, de fecha 12 de junio de 2014, $\mathrm{N}^{\circ}$ ICC-02/11-01/11.

CPI, SCP III, Judgment pursuant to article 74 of the Statute, de fecha 21 de marzo de 2016, $\mathrm{N}^{\circ}$ ICC-01/05-01/08.

CPI, SCP III, Public Redacted Version of "Public Redacted Version of "Decision Pursuant to Article 15 of the Rome Statute on the Authorization of an Investigation into the Situation in the Republic of Burundi”, de fecha 9 de noviembre de 2017, N ICC-01/17-X-9-USExp, 25 October 2017.

CPI, SCP I, Warrant of Arrest for Al Hassan Ag Abdoul Aziz Ag Mohamed Ag Mahmoud, de fecha 27 de marzo de 2018, No ICC-01/12-01/18.

CPI, SCP I, Decision on the Prosecutor's Application for the Issuance of a Warrant of Arrest for Al Hassan Ag Abdoul Aziz Ag Mohamed Ag Mahmoud, de fecha 22 de mayo de 2018, $\mathrm{N}^{\circ}$ ICC-01/12-01/18.

CPI, SCP II, Decision Pursuant to Article 15 of the Rome Statute on the Authorisation of an Investigation into the Situationin the Islamic Republic of Afghanistan, de fecha 12 de abril de 2019, No ICC-02/17.

CPI, SCP IV, Judgment Bosco Ntaganda, de fecha 8 de julio de 2019, N ICC-01/04-02/06.

CPI, SCP I, Rectificatif à la Décision relative à la confirmation des charges portées contre $\mathrm{Al}$ Hassan Ag Abdoul Aziz Ag Mohamed Ag Mahmoud, de fecha 8 de noviembre de 2019, $\mathrm{N}^{\circ}$ ICC-01/12-01/18.

CPI, SCP III, Decision Pursuant to Article 15 of the Rome Statute on the Authorisation of an Investigation into the Situation in the People's Republic of Bangladesh/Republic of the Union of Myanmar, de fecha 14 de noviembre de 2019, N ICC-01/19.

Estatuto de Roma de la CPI, adoptado el 17 de julio de 1998.

Elementos de los Crímenes de la CPI, en el periodo de sesiones de la Asamblea de Estados Partes 3 a 10 de septiembre de 2002 (ICC-ASP/1/3 y Corr. 1), parte II.B y en la Conferencia de Revisión de 31 de mayo a 11 de junio de 2010 (RC/11), parte II.

Estatuto de la Corte Internacional de Justicia, aprobado como anexo de la Carta de Naciones Unidas el 26 de junio de 1945.

Protocolo Adicional I a los Convenios de Ginebra de 1949, relativo a la protección de las víctimas de los conflictos armados internacionales, aprobado el 8 de junio de 1977.

Tribunal Penal Internacional para la ex-Yugoslavia, Judgement, de 12 de junio de 2002, IT-96-23\&IT-96-23/1-A. 
